\title{
Physical Health Outcomes of Conjugal Bereavement: A Psychoneuroendocrine Model of Resilience
}

\author{
Julian C L Lai ${ }^{1 *}$, Xiadong Yue ${ }^{1}$ and Su-Xia $\mathrm{Li}^{2}$
}

${ }^{1}$ Department of Applied Social Studies, City University of Hong Kong, Hong Kong

${ }^{2}$ National Institute on Drug Dependence, Peking University, China

\begin{abstract}
Conjugal bereavement, which is considered as one of the most intense psychosocial stressors, has been studied extensively for the last three decades. However, despite the wealth of data indicating the deleterious consequences of the death of a spouse, the process by which bereavement is translated into ill health and increased mortality remains unclear. Prior research has shown that not all individuals undergoing conjugal bereavement are equally affected. Increased morbidity is more likely to be observed in the young-old group, and those who are not able to cope with grief adaptively. A careful examination of the literature identifies two major issues that have not yet been addressed adequately. The first relates to the elusive physiological mechanisms underlying bereavement-induced morbidity. The second concerns the exclusive focus on vulnerability in prior research, which results in a lack of understanding of the factors facilitating better adjustment. This paper addresses the two issues, and puts forward a psychoneuroendocrine model to inform future research.
\end{abstract}

Keywords: Conjugal bereavement; Hypothalamic-pituitary-adrenal (HPA) axis; Cortisol; cytokines; Wound healing; Resilience

\section{Introduction}

Spousal bereavement is considered as one of the most intense stressors requiring substantial life readjustment [1]. The consequences for physical health of the loss of a spouse have been studied extensively over the last three decades. Summarizing findings from 13 longitudinal studies published since 1999, Stroebe et al. [2] pointed out that an increase in morbidity is consistently observed in the early months of bereavement, and that young-old widowers is the most vulnerable subgroup. The risk of a new or worsened illness increases in the early months after loss, but decreases after six months. Excessive mortality is also observed during the early months after bereavement but decreases over time. The causes of mortality among the bereaved are various, and include suicide. Despite the significance of the aforementioned data, several dimensions of the relationship between conjugal bereavement and physical ill health have received relatively little attention. The first concerns the psychophysiological mechanisms that mediate lossinduced morbidity. Despite three decades' worth of research in this area, the process by which bereavement is translated into physical ill health is still not completely understood. Second, as the existing research has focused exclusively on vulnerability factors, few insights have been gained into the factors that confer resilience on the bereaved. Although it is now widely appreciated that both risk and resilience factors determine the trajectory of adaptation to bereavement, relatively few studies have addressed resilience in this context. I will address these gaps in the literature in the following and propose a psychoneuroendocrine model of resilience to inform future research directions.

\section{Physiological Changes Following Exposure to Chronic Stressors}

The physiological consequences and physical health implications of conjugal bereavement have been studied extensively over the last three decades. This stressor was conceptualized as an intense and chronic stressor inducing detectable changes in the immune and endocrine systems [3]. The physiological impact of conjugal bereavement may follow two major pathways: nonspecific and specific. In response to a stressor, a number of physiological changes are set in motion to allow the individual to cope with the stressor. These major physiological changes are initiated by the activation of the hypothalamic-pituitary-a drenocortical (HPA) axis and the sympathetic-adrenomedullary (SAM) axis [4]. Despite the adaptive nature of these physiological responses, prolonged activation of the aforementioned two axis results in chronic secretion of glucocorticoid and catecholamines. As a variety of immune cells exhibit receptors for cortisol and catecholamines, the chronic secretion of these neuroendocrine products may dysregulate immune function by changing cellular trafficking and proliferation, cytokine secretion, antibody production, and cytolytic activity, among others [5] According to a recent review, the altered physiological state induced by bereavement is characterized by an elevation in cortisol and immune imbalance, which may account for the increase in cardiovascular risk in early bereavement [6]. However, not all individuals respond to the same stressor in an identical way. Superimposed upon the non-specific endocrine and immune changes are a set of specific changes associated with stable individual differences and/or variation in coping with a particular stressor [7]. For instance, there is evidence showing that failure in coping with bereavement results in complicated grief having deleterious endocrinological effects as indexed by dysregulation in the diurnal rhythm of cortisol [8]. Moreover, individuals with a harmavoidant temperament have been shown to be more likely to exhibit dysregulation in endocrine and immune function following spousal bereavement [3].

*Corresponding author: Julian C L Lai, Department of Applied Social Studies, City University of Hong Kong, Hong Kong, Tel: +852 34424306; Fax: +852 34420283; E-mail: ssjulwin@cityu.edu.hk

Received December 23, 2013; Accepted January 29, 2014; Published February 03, 2014

Citation: Lai JCL, Yue X, Li SX (2014) Physical Health Outcomes of Conjugal Bereavement: A Psychoneuroendocrine Model of Resilience. Pharm Anal Acta 5: 284. doi: $10.4172 / 2153-2435.1000284$

Copyright: ( 2014 Lai JCL, et al. This is an open-access article distributed under the terms of the Creative Commons Attribution License, which permits unrestricted use, distribution, and reproduction in any medium, provided the original author and source are credited. 


\section{Physiological Changes during Bereavement}

The findings of a handful of early longitudinal studies substantiated the association between conjugal bereavement and suppressed immunity in the early months of bereavement, as indexed by attenuated blastogenic responses to mitogens during this period [9]. Although these findings have been replicated in a recent study, the physical-health implications of the transient down-regulation of immune function is unclear, as indicated by results of studies examining the impact of immunological changes on physical health in bereaved spouses $[3,10]$. Comparing subgroups of the conjugally bereaved, Zisook et al. [11] found that only widows with major depressive syndromes exhibited impaired immune function as reflected by an attenuation in NK cell activity and blastogenic responses to mitogens. O'Connor et al. [8] have reported that women who experience the loss of a mother or sister and exhibit complicated grief (CG) have a flatter diurnal cortisol slope than their same-sex peers who do not exhibit CG. However, this finding may not be extended in a simple fashion to conjugal bereavement because the trajectory of adaptation varies with the type of lost relationship [2]. A recent study has shown an increased level of proinflammatory cytokines in bereaved spouses in comparison to matched controls [12], but the question of whether the increase in circulating inflammatory markers would actually increase the incidence of inflammatory diseases in the bereaved participants has not been addressed in the study. Taken together, these findings suggest that dysregulation in immune and neuroendocrine function is more accurately predicted by psychological maladjustment to bereavement than by loss per se. However, there is no direct evidence showing that short-term alterations in immune or neuroendocrine function contribute significantly to the excessive morbidity observed in the bereaved.

\section{In Search of a Reliable Health Outcome in Conjugal Bereavement}

One possible explanation for the lack of a relationship observed between changes in immune/endocrine parameters and health status is that prior studies relied heavily on self-reported measures of health outcomes whose links to specific immune or endocrine parameters are unclear. In order to identify and examine the neuroendocrine mechanisms mediating the health consequences of conjugal bereavement, a new paradigm is needed to link loss-induced physiological changes to an objective health outcome that can be monitored and assessed longitudinally. Recent advances in woundhealing research have shown clearly that the healing of experimental cutaneous wounds is a reliable and valid index of health outcome in the context of chronic stress. Psychosocial factors influence the healing of cutaneous wounds in animals as well as humans [13]. Exposure to everyday stressors [14] and/or chronic stressors tends to delay healing whereas social support has been shown to promote healing [15]. Crucially, as pointed out by Broadbent and Koschwanez [16], wound healing is itself a health outcome, and thus provides more direct and valid information than other biomarkers of health. As the immunological and endocrine mechanisms mediating wound healing have been clearly delineated [17], wound healing enables researchers to study both health outcomes and their mediating physiological mechanisms at the same time. In addition, the progress in the healing of experimental cutaneous wounds can be assessed reliably by using ultrasound scans that allows researchers to measure accurately the change in wound diameter over time [18].

It is now known that cortisol [19], the end product of the hypothalamic-pituitary-adrenal (HPA) axis, and proinflammatory cytokines [20] are interactively involved in wound healing in humans. Psychological stress delays wound healing by shifting the cytokine balance towards a Th2 profile that is associated with antibody production rather than cell-mediated immune activities [13].The HPA axis is one of the major pathways through which this effect is produced [5]. The stress-induced increase in the production of cortisol causes a decrease in the local production of proinflammatory cytokines, thereby attenuates the initial inflammatory phase of the repair process [20]. Impairment in the early response will disrupt the entire healing process. However, despite the apparent superiority of wound healing to other biomarkers/measures of health, this measure has not been adopted in research on spousal bereavement.

\section{Control of Cortisol Secretory Activity}

One of the major challenges of studying the mediating effect of cortisol on stress and health outcomes is the establishment of reliable indices of cortisol secretory activity. The release of cortisol into the blood stream is a result of a cascade initiated in the hypothalamus. Specifically, the corticotropin-releasing-hormone $(\mathrm{CRH})$ is synthesized in and released by the paraventricular nucleus (PVN) of the hypothalamus into the portal blood circulation. When the $\mathrm{CRH}$ reaches the anterior pituitary gland, it stimulates the secretion of adrenocorticotropic hormone (ACTH). ACTH enters the systemic blood circulation, and stimulates the synthesis and secretion of glucocorticoids, with cortisol being the main glucocorticoid in humans [21]. Cortisol has profound effects on glucose metabolism, helps to make fat available for energy, and modulate the immune responses, among other physiological functions that it regulates [22]. Exposure to psychosocial stress intensifies the activity of the HPA axis, which results in an increase in the secretion of cortisol to maintain homeostasis. This increase in cortisol secretion is controlled by a negative feedback mechanism which detects increased cortisol level by receptors located in multiple brain regions such as the hypothalamus, the hippocampus, and the pituitary gland $[22,23]$. Although the cortisol response to psychosocial stressors is adaptive, chronic elevation of this hormone has been shown to lead to a number of pathological conditions such as psychiatric disorders, cardiovascular diseases, and type 2 diabetes [24].

\section{Measuring Cortisol Secretory Activity}

The basal level of HPA axis activity follows a well-documented diurnal rhythm with several episodes of high-amplitude secretion. Cortisol secretion peaks within 60 minutes after waking and declines thereafter until reaching the nadir around midnight. Recent evidence suggests that the Cortisol Awakening Response (CAR), which is marked by an increase from $50 \%$ to $150 \%$ within the first 30 minutes after waking up in the morning, is a reliable marker of HPA axis activity [25]. This distinct component of the cortisol diurnal rhythm has a salient genetic component because CAR concordance in MZ twins is statistically greater than that observed in DZ twins [26]. An absence or accentuation of the CAR is associated with various psychopathological conditions or adverse outcomes such as chronic stress [27], and depressive symptomatology [28]. The CAR can be divided into two separate components: the area under the curve with respect to ground $\left(\mathrm{AUC}_{\mathrm{G}}\right)$ and the area under the curve with respect to increase (AUC $\left.\mathrm{I}_{\mathrm{I}}\right)$ [29]. The former captures the overall concentration whereas the latter indexes the increase from after post-awakening. However, a number of researchers have argued that CAR should be used restrictively to refer to the increase in cortisol from after awakening and operationalized by the $\mathrm{AUC}_{\mathrm{I}}$ or similar indices [22]. 
Another reliable marker of the activity of the HPA axis is the diurnal variation or decline which has been operationalized as a descent of cortisol levels measured at different times over the day [30,31]. As mentioned earlier, the cortisol diurnal pattern is characterized by a gradual descent from high levels in the post-awakening period to lower levels in the evening. The diurnal decline has been operationalized in prior research as a decrease in the cortisol levels measured at different times during the day [30]. For example, the diurnal slope which is computed by dividing the difference between the cortisol level at 30 minutes and 12 hours post-awakening by the time interval between these two samples [32]. A flatter profile has been observed among people with depression [33], PTSD [34], and under severe parenting stress [35].

\section{Vulnerability versus Resilience in Conjugal Bereavement}

The exclusive focus in prior research on vulnerability or risk factors has precluded an adequate understanding of factors that confer resilience to the spousally bereaved. Although studies have shown complicated grief and prolonged dysphoric mood to be major factors predicting subsequent morbidity [2,3], a substantial portion (almost $50 \%)$ of the bereaved were resilient and did not exhibit increased grief and depressive symptoms [36]. Although the antecedents of maladaptation to bereavement have been extensively studied, relatively little is known about the factors leading to resilience in the bereaved, or their effects on physical health.

The risk factors examined in prior studies include a number of situational, socio-demographic, and psychosocial factors [2]. Sudden death of a spouse is associated with more negative impact than an expected death. Higher pre-loss care giving strain is also associated with more negative impact of subsequent loss due primarily to neglect of one's own health. In terms of sociodemographic variables, socioeconomic status does not affect adjustment to bereavement. However, widowers and the younger are more vulnerable. Factors pertaining to the pre-loss relationship have also been studied but the findings are mixed [20]. Concurrent stressors such as financial hardship and drop in economic resources tend to complicate the adjustment and make the bereaved more vulnerable.

On the other hand, a number of psychosocial resources have been shown to confer resilience to the negative health effects of bereavement. In particular, higher levels of optimism and self-esteem, and secure attachment styles are associated with better adjustment [2]. Psychological resilience, which refers to the ability to emerge from stress or adversity, has also been found to predict better daily adjustment in widows [37]. As shown by Tugade and Fredrickson [38], individuals with high levels in psychological resilience exhibit faster cardiovascular recovery in response to laboratory stressors. Tugade and Fredrickson [38] argued that this ability to bounce back physiologically faster from acute stressors may lead to better physical well-being in resilient individuals in the long run.

With respect to the effect of psychological resilience on cortisol, the number of studies demonstrating the sensitivity of cortisol to factors closely associated with psychological resilience is growing $[23,39]$. Various indices of the diurnal cycle of salivary cortisol have been reported to be associated with optimism [40,41], positive affect [42], humor [43], social network characteristics [44], and positive wellbeing [45]. A recent study has also demonstrated a positive association

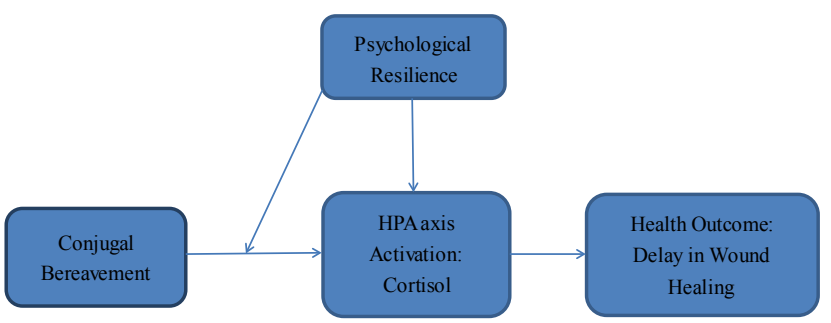

Figure 1: Impact of Psychological Resilience on Wound Healing in the Bereaved. Bereavement leads to poor health outcome (e.g., delay in wound healing) via chronic activation of the HPA axis and the resulting dysregulation in cortisol secretion. Psychological resilience is conceptualized as the key factor modulating this pathogenic pathway by (1) mitigating the negative impact of bereavement on activity of the HPA axis and (2) contributing to maintenance of a normal healthy diurnal rhythm of cortisol.

between optimism, a key component of psychological resilience [45], and rate of wound healing in healthy men [19], thus providing support for the role cortisol possibly plays in mediating the relationship between psychological resilience and wound healing.

\section{Conclusion}

\section{A Psychoneuroendocrine Model of resilience in conjugal bereavement}

Integrating findings reviewed in the previous sections, a newly developed conceptual framework is formulated to guide research on the adjustment to conjugal bereavement (Figure 1). As researchers have consistently demonstrated the positive health effects of psychological resilience, and its recruitment of crucial personal and interpersonal resources that promote successful adaptation to a wide range of stressors [38], this construct can be hypothesized as a key predictor of wound healing among bereaved spouses via its direct and indirect effect on cortisol secretion. Dysregulation in diurnal cortisol rhythm is conceptualized as the physiological mediator because of the prime role of neuroendocrine processes in wound healing, and its association with multiple health pathways compatible with the diversity of causes of morbidity and mortality in bereavement [46].

The significance of the framework lies in its ability to generate research having significant empirical and clinical implications. As bereavement serves as a clearly defined longitudinal model of stress, the framework will lead to studies that shed light on the mechanisms whereby chronic stress in general is translated into ill health. In addition, the framework also facilitates research to pinpoint the principal physiological and psychological mechanisms that explain morbidity in bereavement, thus leading to the formulation of effective pharmacological and psychological interventions for the bereaved.

\section{References}

1. Holmes TH, Rahe RH (1967) The Social Readjustment Rating Scale. J Psychosom Res 11: 213-218.

2. Stroebe M, Schut H, Stroebe W (2007) Health outcomes of bereavement Lancet 370: 1960-1973.

3. Gerra G, Monti D, Panerai AE, Sacerdote P, Anderlini R, et al. (2003) Longterm immune-endocrine effects of bereavement: relationships with anxiety levels and mood. Psychiatry Res 121: 145-158.

4. SELYE H (1950) Stress and the general adaptation syndrome. Br Med J 1 1383-1392.

5. Padgett DA, Glaser R (2003) How stress influences the immune response Trends Immunol 24: 444-448.

6. Buckley T, McKinley S, Tofler G, Bartrop R (2010) Cardiovascular risk in early 
Citation: Lai JCL, Yue X, Li SX (2014) Physical Health Outcomes of Conjugal Bereavement: A Psychoneuroendocrine Model of Resilience. Pharm Anal Acta 5: 284. doi: 10.4172/2153-2435.1000284

bereavement: a literature review and proposed mechanisms. Int J Nurs Stud 47: $229-238$

7. Denson TF, Spanovic M, Miller N (2009) Cognitive appraisals and emotions predict cortisol and immune responses: A meta-analysis of acute laboratory social stressors and emotion inductions. Psychological Bulletin 135: 823-853.

8. O'Connor MF, Wellisch DK, Stanton AL, Olmstead R, Irwin MR (2012) Diurna cortisol in Complicated and Non-Complicated Grief: slope differences across the day. Psychoneuroendocrinology 37: 725-728.

9. Bartrop RW, Luckhurst E, Lazarus L, Kiloh LG, Penny R (1977) Depressed lymphocyte function after bereavement. Lancet 1: 834-836.

10. Lindstrøm TC (1997) Immunity and somatic health in bereavement: A prospective study of 39 Norwegian widows. Omega: Journal of Death and Dying 35: 231-241.

11. Zisook S, Shuchter SR, Irwin M, Darko DF, Sledge P, et al. (1994) Bereavement depression, and immune function. Psychiatry Res 52: 1-10.

12. Schultze-Florey CR, Martìnez-Maza O, Magpantay L, Breen EC, Irwin MR, et al. (2012) When grief makes you sick: Bereavement induced systematic inflammation is a question of genotype. Brain Behav Immun 26: 1066-1071.

13. Godbout JP, Glaser R (2006) Stress-induced immune dysregulation: implications for wound healing, infectious disease and cancer. J Neuroimmune Pharmacol 1: 421-427.

14. Kiecolt-Glaser JK (2006) Social support buffers stress-induced impairments in wound healing. Brain Behav Immun 20: e1-e16.

15. Gouin JP, Carter CS, Pournajafi-Nazarloo H, Glaser R, Malarkey WB, et al. (2010) Marital behavior, oxytocin, vasopressin, and wound healing. Psychoneuroendocrinology 35: 1082-1090.

16. Broadbent E, Koschwanez HE (2012) The psychology of wound healing. Curr Opin Psychiatry 25: 135-140.

17. Dyson M (1997) Advances in wound healing physiology: the comparative perspective. Veterinary Dermatology 8: 227-233.

18. Dyson M, Moodley S, Verjee L, Verling W, Weinman J, et al. (2003) Wound healing assessment using $20 \mathrm{MHz}$ ultrasound and photography. Skin Res Technol 9: 116-121.

19. Ebrecht M, Hextall J, Kirtley LG, Taylor A, Dyson M, et al. (2004) Perceived stress and cortisol levels predict speed of wound healing in healthy male adults. Psychoneuroendocrinology 29: 798-809.

20. Glaser R, Kiecolt-Glaser JK, Marucha PT, MacCallum RC, Laskowski BF, et al. (1999) Stress-related changes in proinflammatory cytokine production in wounds. Arch Gen Psychiatry 56: 450-456

21. Miller DB, O'Callaghan JP (2002) Neuroendocrine aspects of the response to stress. Metabolism 51: 5-10.

22. Smyth N, Clow A, Thorn L, Hucklebridge F, Evans $P$ (2013) Delays of 5-15 min between awakening and the start of saliva sampling matter in assessment of the cortisol awakening response. Psychoneuroendocrinology 38: 1476-1483.

23. Fries E, Dettenborn L, Kirschbaum C (2009) The cortisol awakening response (CAR): facts and future directions. Int J Psychophysiol 72: 67-73.

24. Miller GE, Chen E, Zhou ES (2007) If it goes up, must it come down? Chronic stress and the hypothalamic-pituitary-adrenocortical axis in humans. Psychol Bull 133: 25-45.

25. Clow A, Thorn L, Evans P, Hucklebridge F (2004) The awakening cortiso response: methodological issues and significance. Stress 7: 29-37.

26. Wüst S, Federenko I, Hellhammer DH, Kirschbaum C (2000) Genetic factors, perceived chronic stress, and the free cortisol response to awakening. Psychoneuroendocrinology 25: 707-720.

27. Schlotz W, Hellhammer J, Schulz P, Stone AA (2004) Perceived work overloadand chronic worrying predict weekend- weekday differences in the cortisol awakening response. Psychosomatic Medicine 66: 207-214

28. Pruessner M, Hellhammer DH, Pruessner JC, Lupien SJ (2003) Self-reported depressive symptoms and stress levels in healthy young men: associations with the cortisol response to awakening. Psychosom Med 65: 92-99.

29. Pruessner JC, Kirschbaum C, Meinlschmidt G, Hellhammer D (2003) Two formulas for computation of the area under the curve represent measures of total hormone concentration versus time-dependent change. Psychoneuroendocrinology 28: 916-931.
30. Edwards S, Hucklebridge F, Clow A, Evans P (2003) Components of the diurnal cortisol cycle in relation to upper respiratory symptoms and perceived stress. Psychosomatic Medicine65: 320-327.

31. Kraemer HC, Giese-Davis J, Yutsis M, O'Hara R, Neri E, et al. (2006) Design decisions to optimize reliability of daytime cortisol slopes in an older population. Am J Geriatr Psychiatry 14: 325-333.

32. Adam EK, Kumari M (2009) Assessing salivary cortisol in large-scale epidemiological research. Psychoneuroendocrinology 34: 1423-1436.

33. Hsiao FH, Yang TT, Ho RTH, Jow GM, Ng SM, et al. (2010) The self-perceived symptom distress and health-related conditions associated with morning to evening diurnal cortisol patterns in outpatients with major depressive disorder. Psychoneuroendocrinology 35: 503-515.

34. Yehuda R, Golier JA, Harvey PD, Stavitsky K, Kaufman S, et al. (2005) Relationship between cortisol and age-related memory impairments in Holocaust survivors with PTSD. Psychoneuroendocrinology 30: 678-687.

35. Seltzer MM, Almeida DM, Greenberg JS, Savla J, Stawski RS, et al. (2009) Psychosocial and biological markers of daily lives of midlife parents of children with disabilities. J Health Soc Behav 50: 1-15.

36. Bonanno GA, Wortman CB, Lehman DR, Tweed RG, Haring M, et al. (2002) Resilience to loss and chronic grief: a prospective study from preloss to 18-months postloss. J Pers Soc Psychol 83: 1150-1164.

37. Ong AD, Bergeman CS, Bisconti TL, Wallace KA (2006) Psychologica resilience, positive emotions, and successful adaptation to stress in later life. J Pers Soc Psychol 91: 730-749.

38. Tugade MM, Fredrickson BL (2004) Resilient individuals use positive emotions to bounce back from negative emotional experiences. J Pers Soc Psychol 86: 320-333.

39. Chida Y, Steptoe A (2009) Cortisol awakening response and psychosocial factors: a systematic review and meta-analysis. Biol Psychol 80: 265-278.

40. Endrighi R, Hamer M, Steptoe A (2011) Associations of trait optimism with diurnal neuroendocrine activity, cortisol responses to mental stress, and subjective stress measures in healthy men and women. Psychosomatic Medicine 73: 672-678.

41. Lai JC, Evans PD, Ng SH, Chong AM, Siu OT, et al. (2005) Optimism, positive affectivity, and salivary cortisol. Br J Health Psychol 10: 467-484.

42. Steptoe A, Gibson EL, Hamer M, Wardle J (2007) Neuroendocrine and cardiovascular correlates of positive affect measured by ecological momentary assessment and by questionnaire. Psychoneuroendocrinology 32: 56-64.

43. Lai JC, Chong AM, Siu OT, Evans P, Chan CL, et al. (2010) Humor attenuates the cortisol awakening response in healthy older men. Biol Psychol 84: 375380

44. Lai JC, Chong AM, Siu OT, Evans P, Chan CL, et al. (2012) Socia network characteristics and salivary cortisol in healthy older people. ScientificWorldJournal 2012: 929067.

45. Evans P, Forte D, Jacobs C, Fredhoi C, Aitchison E, et al. (2007) Cortiso secretory activity in older people in relation to positive and negative well-being Psychoneuroendocrinology 32: 922-930.

46. Elwert F, Christakis NA (2008) The effect of widowhood on mortality by the causes of death of both spouses. Am J Public Health 98: 2092-2098. 